\title{
Simulación en educación para ciencias de la salud: ¿Qué calidad hemos alcanzado en Chile?
}

\author{
Eliana Escudero ${ }^{1,2}$, Carlos Fuentes ${ }^{3}$, Matías González ${ }^{4}$, Marcia Corvetto ${ }^{3 *}$
}

Resumen: Introducción: en Chile, la simulación está siendo ampliamente utilizada en educación para ciencias de la salud; sin embargo, la calidad con la que se ha estado implementando es desconocida. Objetivo: describir la calidad de los centros de simulación en Chile de acuerdo al cumplimiento de los estándares de acreditación internacional propuestos por la Society for Simulation in Healthcare (SSH). Métodos: con la aprobación del comité de ética institucional, se diseñó una encuesta basada en los estándares de Acreditación para los Programas de Simulación (Acreditation Standards of Healthcare Simulation Programs) de la SSH. Este instrumento se envió vía e-mail a los 17 Centros de Simulación pertenecientes a universidades del país y fue procesado anónimamente. Resultados: 13 centros de simulación aceptaron participar (tasa de respuesta de 76,47 \%). El 92,3\% cuenta con una misión del programa, el 53,8\% tiene un director con horario exclusivo y la mayoría cuentan con la infraestructura necesaria. Son muy frecuentes la simulación para habilidades, alta fidelidad y poco frecuentes los pacientes estandarizados y la simulación virtual. La implementación de estrategias de mejoramiento continuo no es evidente en todos los centros y no se están desarrollando sistemáticamente proyectos de investigación. Conclusiones: se ha realizado la primera encuesta sobre el cumplimiento de los estándares de calidad definidos por la SSH, en los Centros de Simulación universitarios en Chile. Globalmente, existe un alto grado de cumplimiento de la mayoría de los ítems; sin embargo, la evaluación e investigación son áreas que requieren desarrollo.

Abstract: Introduction: In Chile, simulation-based training has being increasingly used as part of educational programs for health sciences; however, the quality with which this methodology has been used is unknown. Objective: To describe the quality of simulation centers in Chile, based on its compliance with the Acreditation Standards of Healthcare Simulation Programs proposed by the Society for Simulation in Healthcare (SSH). Methods: After institutional ethics committee approval, a survey based on the Accreditation Standards for Simulation Programs of the SSH was designed. The instrument was sent to the 17 simulation university centers in the country via e-mail and was processed anonymously. Results: 13 university simulation centers agreed to participate (response rate of 76.47\%). $92.3 \%$ have a mission program, 53.8\% have a director with exclusive schedule and most centers have the necessary infrastructure. Simulation for skills and high fidelity are common; standardized patients and virtual simulation are scarce. Implementation of strategies for continuous improvement is not evident in all centers and research is not being systematically developed. Conclusions: This is the first survey about the quality standard compliance defined by the SSH of Chilean. Overall results shown a high degree of accomplishment of most of the items, however, assessment and research are areas that should be developed.

Palabras clave: Simulación; Simulación de pacientes; Educación médica

Keywords: Simulation; Patient Simulation; Medical Education

Fecha de envío: 17 de 0ctubre de 2016 - Fecha de aceptación: 20 de Diciembre de 2016

\section{Introducción}

En los últimos años ha habido un cambio en el paradigma de la enseñanza de la medicina y sus especialidades (Konia, 2013). Los pacientes están más conscientes de que los estudiantes "practican" con ellos y a su vez los estudiantes reconocen cada vez más su falta de entrenamiento en ciertas habilidades clínicas, lo que sumado a un sistema médico-legal mucho más potente (MINSAL; 2012), hace que el aprender con pacientes reales, sea cada vez menos aceptado (Ziv et al.; 2003).

(1) Escuela de Enfermería. Universidad FinisTerrae. Chile.

(2) Sociedad Chilena de Simulación Clínica y seguridad del paciente.

(3) División de Anestesiología, Escuela de Medicina, Pontificia Universidad Católica de Chile.

(4) División de Anestesiología. Clínica Santa María. Chile.

*Autor de correspondencia: marciacorvetto@gmail.com 
Por su parte, la simulación reproduce situaciones o procedimientos utilizando maniquíes, actores y/o pacientes virtuales con fines de entrenamiento personal y trabajo seguro en equipo (Gaba, 2007), favorece el aprendizaje a través del error, ofrece la posibilidad de repetir cuantas veces sea necesario (Okuda et al.; 2009), promueve el aprendizaje basado en la experiencia (Boulet, 2010)b y ofrece retroalimentación inmediata, reflexión y puesta en práctica sin generar daño (Eppich, 2015).

En este contexto, el desarrollo de la simulación en Chile en los últimos 10 años y en algunos países de Latinoamérica pareciera ser importante y creciente. Se habla de ello en congresos, se crean sociedades de simulación (SOCHISIM; 2016), las universidades hacen grandes inversiones y los proyectos con financiamiento desde el ministerio de salud y educación son más frecuentes. Así mismo, la industria crece y proporciona a menor costo variadas opciones de productos para desarrollar simulación de baja y alta fidelidad (Kunkler, 2006). Sin embargo, aún no hay evidencia disponible del impacto que ha presentado la incorporación de esta metodología en el currículo de las carreras de la salud (Bradley, 2006).

Para que el perfil de los egresados cumpla adecuadamente con lo propuesto en sus respectivos programas, no basta con tener centros de simulación y equipos sofisticados de alta tecnología. Se hace necesario profundizar sobre qué actividades se desarrollan en estos centros, cómo se lleva a cabo la docencia, qué apoyo institucional existe y quiénes están a cargo; en otras palabras, con qué estándares de calidad se desarrollan estos proyectos pedagógicos; solo esta información podría dar cuenta del verdadero impacto que tiene la simulación sobre las buenas prácticas y los resultados de seguridad alcanzados (Gaba, 2000).

En Chile, la investigación en esta área es escasa y la información de lo que se está llevando a cabo en Educación basada en Simulación para Ciencias de la Salud (EBSCS) se limita a lo que entrega una página web. Hace unos años se publicó al respecto, (Corvetto et al.; 2013) pero solo se entregó información cualitativa y no cuantitativa por lo que ahora es indispensable acercarse a conocer los estándares de calidad con los que se ha venido utilizando esta herramienta pedagógica.

El objetivo de este manuscrito es describir la calidad de la simulación en Chile de acuerdo a la adherencia de los centros de simulación universitarios del país a los estándares internacionales de acreditación propuestos por la Society for Simulation in Healthcare (SSH) y de esta manera, entregar una primera imagen global sobre la forma en la que la estructura de la educación en simulación para ciencias de la salud se ha venido implementado dentro de los programas de formación profesional y técnica multidisciplinar en nuestro país.

\section{Materiales y métodos}

Posterior a la aprobación del comité de ética institucional de la Universidad Finis Terrae con fecha 15 de marzo de 2016, el proyecto fue puesto en marcha bajo dos etapas. La primera correspondió a la construcción y validación del instrumento de investigación, el cual se diseñó a manera de encuesta tipo Likert basada en los criterios de los Estándares de Acreditación para los Programas de Simulación (Acreditation Standards of Healthcare Simulation Programs) propuestos por la SSH (SSH; 2016). Dichos criterios fueron adaptados al español por una traductora certificada y acercados a la realidad nacional por 2 miembros del equipo investigador. La validez de contenido fue realizada bajo consenso con técnica Delphi incluyendo 5 expertos nacionales e internacionales en simulación contactados por vía electrónica. Una vez construido y validado el instrumento, se procedió a su desarrollo electrónico en la plataforma Surveymonkey ${ }^{\circledast}$ (www.surveymonkey.com).

La segunda etapa correspondió a la aplicación de la encuesta. Se consideró únicamente a los 17 centros de simulación pertenecientes a universidades del país que así lo informaban en su página web institucional. La autorización para participar fue solicitada a cada director o jefe de programa a través de una carta.

Por medio de un linkenviado por correo electrónico, cada uno de los participantes debía contestar el total de las preguntas debido a que la encuesta fue diseñada con carácter obligatorio para todas, adicionalmente tuvieron la posibilidad de indicar comentarios adicionales en cada una de ellas.

Todas las respuestas obtenidas fueron de carácter anónimo y el análisis estadístico descriptivo se obtuvo usando la plataforma de análisis de Surveymonkey ${ }^{\circledR}$.

\section{Resultados}

La encuesta fue traducida al español por una traductora certificada. Posteriormente fue revisada y acercada a nuestra realidad por una comisión integrada por 2 personas del equipo investigador expertos en evaluación y simulación. Se seleccionaron un total de 35 preguntas de relevancia para el desarrollo de la Simulación en Chile (Apéndice 1); estas preguntas fueron agrupadas en 7 grandes ítems descritos por la SSH como "Core Standards":

- Misión y Gobernabilidad.

- Organización y Gestión.

- Instalaciones, tecnología, modalidades de simulación y Recursos Humanos.

- $\quad$ Ambiente de aprendizaje.

- Evaluación y Mejoramiento continuo.

- Integridad.

- Seguridad. 
Posteriormente, se realizó la validación de contenido mediante consenso con técnica Delphi, donde los 2 expertos nacionales y los 3 internacionales invitados accedieron a participar. A partir de esta validación, se realizaron pequeñas modificaciones que no implicaron un cambio sustancial en el instrumento.

Posteriormente, se confeccionó una lista de centros de simulación de acuerdo a los criterios de inclusión generando un universo de 17; de estos, 13 aceptaron participar representando una tasa de respuesta de $76,47 \%$. Los que no respondieron, declararon razones de confidencialidad.

En relación al ítem de Misión y Gobernabilidad, el 92,3\% de los centros cuenta con una Misión del programa o centro de simulación, sin embargo, no todos cuentan con un director con horario exclusivo (solo un 53,8\%); además, es destacable que en el 30,8\% de los encuestados no exista un docente responsable de llevar a cabo esta misión (Figura 1). En el ítem Instalaciones, tecnología, modalidades de simulación y recursos físicos, la mayoría de los centros pareciera contar con la infraestructura necesaria (Figura 2). En relación a las distintas modalidades de simulación, son muy frecuentes la simulación para habilidades, alta fidelidad y poco frecuentes los pacientes estandarizados; menos aún la simulación virtual (Figura 3).

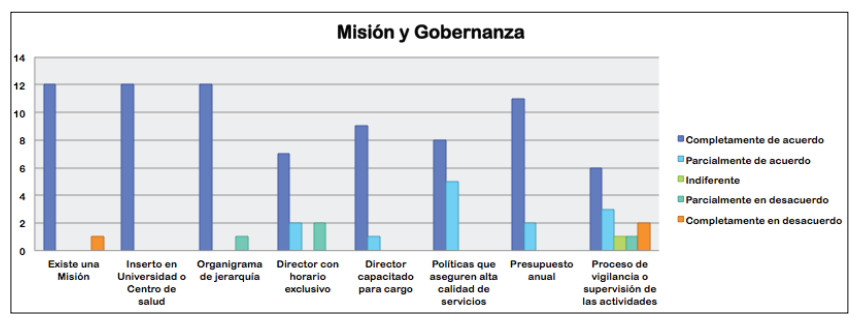

Figura 1. Misión y Gobernanza.

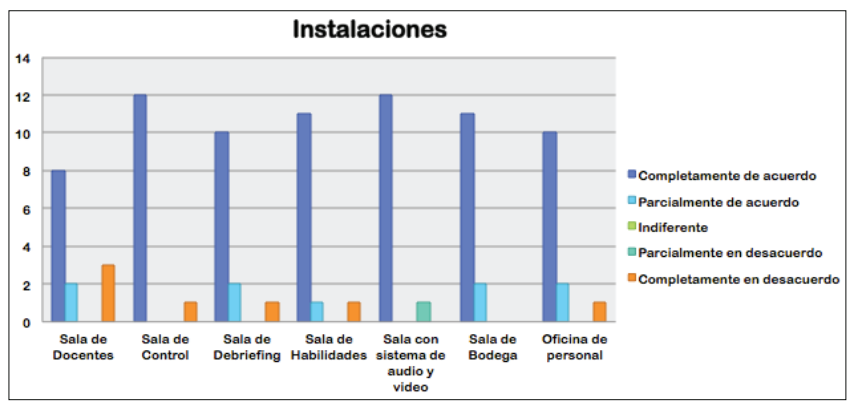

Figura 2. Instalaciones.

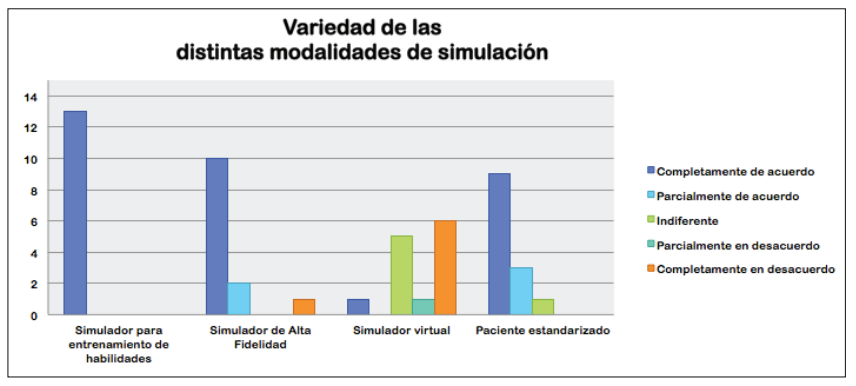

Figura 3. Distintas modalidades de simulación.
En el área de Investigación, los resultados muestran que no se están desarrollando sistemáticamente proyectos de esta índole. Adicionalmente, en el $23,1 \%$ de estos centros, no existe una política de investigación que responda a un comité de ética institucional (Figura 4). Con respecto al ítem Evaluación y Mejoramiento continuo, la implementación de estrategias de mejoramiento a partir de pautas de retroalimentación propia no es evidente en todos los centros. Por último, pese a que la mayoría de los centros realiza actividades de evaluación con simulación, no todos cuentan con un proceso de selección de instrumentos ni de evaluación docente (al menos anual) que persiga asegurar competencias y desarrollo profesional (Figura 5).

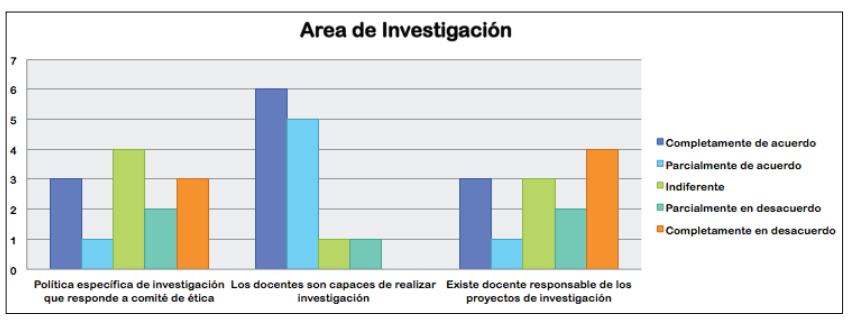

Figura 4. Investigación.

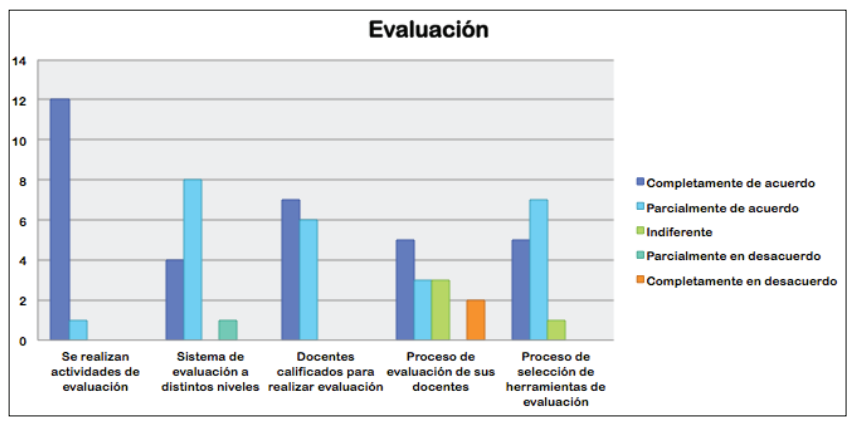

Figura 5. Evaluación.

\section{Discusión}

Se ha realizado la primera encuesta sobre el cumplimiento de los estándares de calidad definidos por la SSH, de los Centros de Simulación en Chile. De forma global, existe un alto grado de cumplimiento de la mayoría de los ítems; sin embargo, los resultados muestran que la tarea de mejorar en pautas de evaluación e investigación es grande.

Nuestros datos muestran una tendencia hacia la implementación de centros universitarios con buena infraestructura, sin embargo, el desarrollo del recurso humano parece no ser prioritario; en este contexto, la mitad de los centros no tienen un director con horario exclusivo y en un tercio de ellos, no hay un docente responsable de llevar a cabo la misión y visión.

La educación médica ha sido clara en señalar la necesidad de innovar incorporando principalmente nuevas metodologías y formas de evaluación validadas (Epstein, 2007). La retroalimentación 
hacia el estudiante debe existir de forma constante, planificada e intencionada y los debriefing, entendidos como una conversación reflexiva sobre las acciones tomadas individualmente y en equipo durante un escenario simulado, deben indagar la metacognición del estudiante (Rudolph et al.; 2008). En este cambio de paradigma, los docentes son esenciales y deben demostrar que califican no solo en lo disciplinar, sino que además cuentan con competencias en esta práctica educativa. La importancia de dar cumplimiento a los perfiles de egresados a través de resultados medibles exigidos para las acreditaciones institucionales, amerita control y seguimiento curricular obligatorios. La antigua forma de hacer docencia, en la que un profesor dictaba una clase magistral, ya no se adapta al modelo educativo actual; por ello, se exige transitar hacia una docencia integrada y centrada en el estudiante, dando espacio al desarrollo de un currículo basado en competencias que incorpore metodologías activas a los procesos de enseñanza y aprendizaje (Smith, 1999); para este propósito, la presencia de un líder en cada centro de simulación, que vele por dichos cumplimientos, parece ser fundamental (Rothgeb, 2008). Adicionalmente el desarrollo continuo de los docentes ha sido descrito en la literatura como un punto crítico para el éxito de un programa de simulación (Rogers et al.; 2015). En esta encuesta, solo la mitad de los centros en Chile refiere tener docentes calificados y su evaluación parece no ser el fuerte. En la actualidad, se ha reconocido a la EBSCS (McGaghie et al.; 2010) como una herramienta valiosa para el proceso cognitivo, que permite la integración, progresión, autoaprendizaje y autorregulación por parte del estudiante (McGaghie et al.; 2011). Es una metodología aceptada para certificación estandarizada de las competencias de las carreras de salud y por ello, no debería ser una actividad aislada que surge de la motivación de un docente, sino que debiese estar plasmada en un plan de estudio, con instancias definidas para que el alumno practique y bajo herramientas sólidas con las que finalmente pueda ser evaluado. Nuestro estudio muestra que los sistemas de evaluación no se están utilizando a distintos niveles y no se está realizando rigurosamente un proceso de selección de los mismos.

En relación a investigación, aparentemente la mayoría de los centros no tiene una política definida que responda a un comité de ética ni docentes a cargo de esta área, sin embargo, llama la atención que en 11 centros hay docentes capacitados para hacer investigación.

Existen varias limitaciones en este estudio. La más importante, es que esta muestra incluyó solo a Centros de simulación Universitarios. Como es evidente, la representatividad y la validez de los datos no es relevante para todos los Centros o"salas" de simulación de nuestro país; sin embargo, teniendo en cuenta las dificultades para lograr buenas tasas de respuesta, estos datos se convierten en evidencia disponible y útil. Otra limitación es la posibilidad de sesgo de los no encuestados. Aunque, una buena tasa de respuesta disminuye el riesgo de sesgo de no respondedores, no podemos saber si los no respondedores hubiesen contestado en la misma proporción que los respondedores. Una tercera, es el sesgo atribuible a enviar la encuesta por correo electrónico ya que solamente los centros con acceso a Internet y utilización regular, tuvieron la oportunidad de responder; sin embargo, este estudio proporciona datos de carácter diagnóstico que pueden ser útiles a futuro para dar inicio a procesos de consenso e integración curricular a nivel nacional en los que se busque unificar pautas docentes de calidad, implementar programas de intercambio de experiencias, generar oportunidades de formación profesional, promover proyectos de investigación y lograr un mayor aprovechamiento de los recursos físicos y humanos de los que disponen los centros de simulación universitarios de nuestro país.

Vale la pena mencionar que el desarrollo de los estándares de educación en simulación se viene abordando desde hace unos años y es probablemente el proyecto Simulation Innovation Resource Center de la organización norteamericana, National League for Nursing (NLS; 2007), el que planteó la primera discusión y propuesta al respecto. Este fue conformando por una comunidad de enfermeras educadoras que trabajó para establecer criterios de evaluación, docencia y creación de centros de simulación, con una comisión de 9 enfermeras norteamericanas y 8 provenientes de Australia, Canadá, Chile, China, Escocia, Japón y Noruega. Después de unos años de trabajo, proporcionaron una serie de cursos a los que se puede acceder actualmente en forma virtual, facilitando el aprendizaje y la preparación de los educadores. En relación a este último punto, nuestro país ofrece actualmente a los interesados en EBSCS, la posibilidad de cursar un diplomado en la universidad Finis Terrae o bien realizar formalmente el curso de instructor en simulación impartido anualmente por el equipo docente del Institute for medical simulation (IMS) de la universidad de Harvard bajo convenio con el Hospital Virtual de Valdecilla (Santander; España) en las dependencias del centro de simulación de la Pontifica universidad Católica de Chile.

Finalmente, con respecto a la evaluación de la calidad de la simulación; en el año 2010, la SSH inició un proyecto de acreditación de educadores y programas cuyo fin es la expansión de la calidad de la educación con simulación clínica y cuyo detalle puede ser revisado en la página web de esta organización (SSH; 2016). Para el 2017, la SSH promete entregar nuevos criterios acuciosamente revisados en los que la Sociedad Chilena de Simulación Clínica y Seguridad del Paciente (SOCHISIM), como una de las entidades multidisciplinares afiliadas y colaboradoras que reúne a profesionales de la salud involucrados en EBSCS, también participó. Los estándares son exigentes y complejos 
de aplicar en nuestra realidad; dan esencial importancia al proyecto pedagógico y calidad docente y no a la inversión masiva en tecnología; así mismo, promueven la investigación y el trabajo en red como claves para calificar adecuadamente, puntos en los que aún existe un evidente grado de subdesarrollo en Latino América; sin embargo, serán estas directrices la mejor referencia disponible para desarrollar con calidad el trabajo que la educación en ciencias de la salud requiere en Chile.

\section{Referencias}

Boulet JR, Murray DJ. Simulation-based assessment in anesthesiology: requirements for practical implementation. Anesthesiology 112, 1041-52.

Bradley P. The history of simulation in medical education and possible future directions. Med Educ 40, 254-62.

Corvetto M, Bravo MP, Montana R, Utili F, Escudero E, Boza C, Varas J, Dagnino J. Simulation in medical education: a synopsis. Rev Med Chil 141, 70-9.

Eppich W, Cheng A. Promoting Excellence and Reflective Learning in Simulation (PEARLS): development and rationale for a blended approach to health care simulation debriefing. Simul Healthc 10, 106-15.

Epstein RM. Assessment in medical education. N Engl J Med 356, 387-96.

Gaba DM. Anaesthesiology as a model for patient safety in health care. BMJ 320, 785-8.

Gaba DM. The Future Vision of Simulation in Healthcare. Simulation in Healthcare 2, 126-35.

Konia M, Yao A. Simulation-a new educational paradigm? Journal of biomedical research $27,75-80$.

Kunkler K. The role of medical simulation: an overview. The International Journal of Medical Robotics and Computer Assisted Surgery 2, 203-10.

McGaghie W, Issenberg S, Petrusa E, Scalese R. A critical review of simulation-based medical education research: 2003-2009. Medical Education 44, 50-63.
McGaghie WC, Issenberg SB, Cohen ER, Barsuk JH, Wayne DB. Does simulation based medical education with deliberate practice yield better results than traditional clinical education? A meta-analytic comparative review of the evidence. Acad Med 86, 706-11.

Ministerio de Salud; Subsecretaría de Salud Pública. (2012). LEY NúM. 20.584; Regula los derechos y deberes que tienen las personas en relación con acciones vinculadas a atención en salud. Accedido en: https://www.leychile.cl/Navegar?idNorma=1039348\&idVersion=2012-10-01 el (01 de Diciembre de 2016)

National League for Nursing; Simulation Innovation Resource Center (2007); A collaborative Alliance to advance simulation in nursing education. Accedido en: http://sirc.nln.org/mod/resource/view. php?id=347. El (01 de Diciembre de 2016).

Okuda Y, Bryson EO, DeMaria S, Jr., Jacobson L, Quinones J, Shen $B$, Levine Al. The utility of simulation in medical education: what is the evidence? Mt Sinai J Med 76, 330-43.

Rogers DA, Peterson DT, Ponce BA, White ML, Porterfield JR, Jr. Simulation and Faculty Development. Surg Clin North Am 95, 729-37.

Rothgeb MK. Creating a nursing simulation laboratory: a literature review. The Journal of nursing education 47, 489-94.

Rudolph JW, Simon R, Raemer DB, Eppich WJ. Debriefing as formative assessment: closing performance gaps in medical education. Acad Emerg Med 15, 1010-6.

Sociedad Chilena de Simulación y Seguridad del Paciente. Accedido en: http://www.sochisim.cl el (01 de Diciembre de 2016).

Society for Simulation in Healthcare (2016). Accreditation Standards for Simulation Programs. Accedido en: http://www.ssih. org/Accreditation/Full-Accreditation el (01 de Diciembre de 2016).

Smith SR. AMEE guide No. 14: Outcome-based education: Part 2-Planning, implementing and evaluating a competency-based curriculum. Medical Teacher 21, 15-22.

Ziv A, Wolpe PR, Small SD, Glick S. Simulation-based medical education: an ethical imperative. Acad Med 78, 783-8. 